\title{
Histology AS PROGNOSTIC FACTOR IN EARLY-STAGE CERVICAL CARCINOMA. EXPERIENCE IN A THIRD-LEVEL INSTITUTION
}

\author{
Salim Abraham Barquet-Muñoz, Efraín Cruz-Rodríguez, David Francisco Cantú De León*, \\ David Isla-Ortiz, Gonzalo Montalvo-Esquivel, Luis Alonso Herrera-Montalvo, \\ Carlos Pérez-Plasencia, Delia Pérez-Montiel and Ángel Herrera-Gómez
}

Department of Gynecologic Oncology, Instituto Nacional de Cancerología, Mexico City, Mexico

\begin{abstract}
Background: Cervical carcinoma (CC) is one of the most frequent neoplasms, especially in developing countries. The most common histopathological type is squamous cell carcinoma (SCC), followed by adenocarcinoma (AC) and adenosquamous carcinoma (ASC). Prognosis according to histological type is controversial. Objective: The objective of this study is to describe and compare the prognoses of the most common histologies of CC in the early stages. Materials and Methods: We reviewed records of patients attended at the Instituto Nacional de Cancerología of Mexico with CC surgically treated Stages IA2-IB1 and IIA1, including the histological types SCC, AC, and ASC. Patients who had another malignant neoplasm, cervical cancer in situ, locally advanced neoplasm, and metastatic neoplasm were excluded from the study. A descriptive and comparative analysis was conducted. Overall survival (OS) and disease-free period were calculated for each histological type with the Kaplan-Meier method and were compared with the log-rank test. Results: A total of 202 records were obtained, of which 131 (64.9\%) had SCC, 57 (28.2\%) AC, and 14 (6.9\%) ASC. The 5-year DFS was $94.4 \%$ for SCC, 98.1\% for AC, and 92.3\% for ASC, without a statistically significant difference $(p=0.55)$. The 5-year OS for SCC was $97.9 \%$, for AC was $97.8 \%$, and for ASC was $100 \%$, without a statistically significant difference $(p=0.702)$. Conclusions: DFS and OS did not differ between the most common histological types of CC at the early stages.
\end{abstract}

Key words: Cervical carcinoma. Squamous cell carcinoma. Adenocarcinoma. Adenosquamous carcinoma.

\section{INTRODUCTION}

Cervical carcinoma (CC) is the fourth most common cancer in women worldwide, with a global incidence of 527,624 new cases and a global mortality of 265,672 cases in $2012^{1}$. In total, $85 \%$ of cases occur in developing countries. In Mexico, CC is the second most common cancer in women after breast cancer,

\section{Corresponding author:}

*David Francisco Cantú de León

Clinical Research Instituto Nacional de Cancerología

Av. San Fernando, 22

Col. Sección XVI, Del. Tlalpan

C.P. 14080, Ciudad de México, México

E-mail: dcantude@yahoo.com with annual incidence and mortality of 13,960 and 4769 cases, respectively ${ }^{1}$. In $70 \%$ of cases, squamous cell carcinoma (SCC) is the most common histological type, followed by adenocarcinoma (AC) in 15-20\% 2,3 .

Prognosis by histologic type in CC is controversial. Currently, CC patients with $\mathrm{AC}$ and adenosquamous carcinoma (ASC) histologies receive the same
Received for publication: 11-01-2017

Approved for publication: 17-06-2017

doi: $10.24875 / R I C .17002143$ 
treatment as those with $\mathrm{SCC}^{4}$. However, the studies conducted have only been retrospective, and these analyzed CC prognoses according to histology in patients treated surgically or with radiotherapy $(\mathrm{RT})^{5-11}$. Lee et al. and other researchers have shown that patients with early-stage AC who are treated surgically have a very poor prognosis ${ }^{8}$. However, other investigators have been unable to find this difference in survival between distinct histologic types ${ }^{12}$.

The objective of our study was to analyze the prognosis of patients with the most common histological types of early-stage CC who are surgically treated at the National Cancer Institute (Instituto Nacional de Cancerología, INCan) of Mexico.

\section{MATERIALS AND METHODS}

\section{Patients}

The clinical records of patients with CC treated between January 2005 and December 2013 were reviewed. Patients were included if they had CC in clinical stages from IA2 to IB1 and IIA1 and were surgically treated based on the 2009 International Federation of Gynecology and Obstetrics criteria or the AJCC Staging System $7^{\text {th }}$ edition. The histologies included SCC, AC, and ASC and were determined using the World Health Organization classification system ${ }^{13}$. Patients were excluded if they had another malignant neoplasm, CC in situ, locally advanced and metastatic neoplasms, double primary neoplasms, or incomplete clinical records.

This study was reviewed and approved by the Committee of Investigation and Ethics of the National Institute of Cancerology of Mexico (approval number $\mathrm{Cl} / 216 / 15)$ and adhered to the statutes of the Declaration of Helsinki.

Of the patients selected, all underwent radical hysterectomy $(\mathrm{RH})$ and bilateral pelvic lymphadenectomy (BPL) after they were categorized by risk group based on the pathology reports. The high-risk group included patients with positive pelvic lymph nodes, parametrial invasion, and positive vaginal margins. The intermediate-risk group included patients with a depth of stromal invasion more than $1 / 3$ the cervical wall, lymphovascular space invasion (LVSI), or a tumor diameter larger than $4 \mathrm{~cm}$. Patients in the low-risk group were those not included in either of the previous groups and who did not receive adjuvant treatment. Patients with one or more high-risk factors received adjuvant treatment with concomitant chemotherapy and RT (Chem/ $\mathrm{RT}$ ) and brachytherapy (BT); patients with one or more intermediate-risk factors were only administered RT and BT. RT consisted of 1.8 Gy on days $1-5$ of each week, for a total of 28 fractions, with a total external radiation dose of $50.4 \mathrm{~Gy}$ to the conventional pelvic fields. BT was administered at a high-rate or low-rate dose, depending on the preference of the radiotherapist. The concomitant chemotherapy schemes were cisplatin- or gemcitabine-based, and 4-6 cycles were offered. The chemotherapy agent and scheme were selected based on the medical oncologist's preference. Patients attended a follow-up visit approximately every 3-4 months for the first 2 years, every 6 months for the following 3 years, and annually thereafter.

The following variables were analyzed: Age, body mass index, clinical stage, type of $\mathrm{RH}$, number of resected lymph nodes, adjuvant treatment (when administered), and follow-up. The pathology report included tumor grade, tumor size, stromal invasion, LVSI, parametrial involvement, lymph node invasion, and margin status. The different histologies are defined as follows: SCC: Invasive epithelial tumor composed by sheet-like, cords, and/or nest with polygonal cells with intercellular bridges, nuclear pleomorphism, coarse chromatin, and nucleoli, with or without keratin. AC: Invasive epithelial tumor composed by glands with complex architectural pattern. The neoplastic epithelium shows pseudostratified architecture with enlarged, elongated, and hyperchromatic nuclei. ASC: Invasive epithelial tumor composed of both clearly differentiated AC and SCC components. Recurrence was defined as the appearance of disease 6 months after treatment completion. The overall survival (OS) period was defined as the time between diagnosis of the disease and death or last follow-up visit. The disease-free period (DFP) was defined as the time between the initiation of surveillance and the date of recurrence or the last follow-up visit.

\section{Statistical analysis}

A statistical analysis was conducted including descriptive statistics of each variable. Comparisons between 
variables were performed utilizing Student's t-test for quantitative variables and Chi-squared or Fisher's exact test for qualitative variables. OS and DFP were calculated with the Kaplan-Meier method, and these were compared with log-rank tests. Uni- and multivariate analyses were conducted to analyze risk factors using the Cox method. A value of $p<0.05$ indicated statistically significant. SPSS v22 software was utilized (SPSS, Inc., Chicago, IL, USA).

\section{RESULTS}

A total of 217 clinical records were obtained; of these, 15 were discarded due to incomplete information, resulting in 202 records in the analysis. Table 1 presents the demographic characteristics. There were 131 (64.9\%) patients with SCC, 57 (28.2\%) with AC, and 14 (6.9\%) with ASC histologies. The mean age was 50.8 years for SCC, 46.8 years for AC, and 47.8 years for ASC patients. With respect to clinical stage, $108(82.4 \%)$ of the SCC, 52 (91.2\%) of the AC, and $12(85.7 \%)$ of the ASC cases were Stage $\mathrm{lb} 1 . \mathrm{In}$ regards to surgery, RH Type 2 and RH Type 3 were performed, respectively, in 57 (43.5\%) and 74 (56.5\%) cases in the SCC group, 23 (40.4\%) and 34 (59.6\%) in the AC group, and 3 (21.4\%) and $11(78.6 \%)$ in the ASC group. The mean number of resected lymph nodes was 21.2. In addition, 72 (55\%) SCC patients did not receive any adjuvant treatment, $5(3.8 \%)$ received only BT, 32 (24.4\%) received RT with BT, and 22 (16.8\%) received Chem/RT. Of the AC group, 38 (66.7\%) received no adjuvant management, $2(3.5 \%)$ received BT alone, 9 (15.8\%) received RT with BT, and 8 (14\%) received Chem/RT. Finally, in the ASC histology group, 6 (42.9\%) did not receive adjuvant treatment, $3(21.4 \%)$ received BT alone, and $5(35.7 \%)$ received RT with BT. From the SCC patients, 7 (5.4\%) had experienced recurrence, most of which were local. Only $1(1.8 \%) \mathrm{AC}$ and 1 (7.1\%) ASC patient presented distant recurrence.

Regarding pathologic characteristics, a significant difference was found with respect to the depth of invasion $(p=0.04)$, with the depth being $1 / 3$ in $67(51.1 \%), 2 / 3$ in $33(25.2 \%)$, and $3 / 3$ in

Table 1. Demographic description of patients with early-stage CC who receive a radical hysterectomy

\begin{tabular}{|c|c|c|c|c|}
\hline Variable & $\begin{array}{c}\text { Squamous** } \\
131(64.9)\end{array}$ & $\begin{array}{c}A C^{* *} \\
57(28.2)\end{array}$ & $\begin{array}{c}\text { Adenosquamous** } \\
14(6.9)\end{array}$ & $\mathrm{p}$ \\
\hline Age (years)* & $50.8(11.3)$ & $46.8(10.1)$ & $47.8(12.3)$ & 0.63 \\
\hline \multicolumn{5}{|l|}{$\mathrm{BMI}{ }^{* *}$} \\
\hline$<25$ & $34(26)$ & $19(33.3)$ & $5(35.7)$ & \multirow[t]{3}{*}{0.461} \\
\hline $25-30$ & $62(47.3)$ & $19(33.3)$ & $5(35.7)$ & \\
\hline$>30$ & $35(26.7)$ & $19(33.3)$ & $4(28.6)$ & \\
\hline \multicolumn{5}{|l|}{ Stage** } \\
\hline la2 & $17(13)$ & $4(7)$ & $2(14.3)$ & \multirow[t]{3}{*}{0.534} \\
\hline lb1 & $108(82.4)$ & $52(91.2)$ & $12(85.7)$ & \\
\hline Ila1 & $6(4.6)$ & $1(1.8)$ & $0(0)$ & \\
\hline \multicolumn{5}{|l|}{$\begin{array}{l}\text { Surgical intervention } \\
\text { type** }\end{array}$} \\
\hline $\mathrm{RH} 2$ & $57(43.5)$ & $23(40.4)$ & $3(21.4)$ & \multirow[t]{2}{*}{0.277} \\
\hline $\mathrm{RH} 3$ & $74(56.5)$ & $34(59.6)$ & $11(78.6)$ & \\
\hline Number of lymph nodes* & $19.9(8.3)$ & $20.7(8.4)$ & $22.9(7.6)$ & 0.432 \\
\hline \multicolumn{5}{|l|}{ Adjuvancy** } \\
\hline No & $72(55)$ & $38(66.7)$ & $6(42.9)$ & \multirow[t]{4}{*}{0.024} \\
\hline BT & $5(3.8)$ & $2(3.5)$ & $3(21.4)$ & \\
\hline RT & $32(24.4)$ & $9(15.8)$ & $5(35.7)$ & \\
\hline Chem/RT & $22(16.8)$ & $8(14.0)$ & $0(0)$ & \\
\hline Recurrence** & & & & 0.93 \\
\hline No & $124(94.7)$ & $56(98.2)$ & $13(92.9)$ & \\
\hline Local & $6(4.6)$ & $0(0)$ & $0(0)$ & \\
\hline Regional & $1(0.8)$ & $0(0)$ & $0(0)$ & \\
\hline Distant & $0(0)$ & $1(1.8)$ & $1(7.1)$ & \\
\hline
\end{tabular}

*Mean (SD)

${ }^{* *}$ Absolute frequency (percentage).

BMI: body mass index; RH2: radical hysterectomy Type 2, RH3: radical hysterectomy Type 3; BT: brachytherapy; RT: external radiotherapy with BT; QT/RT: chemotherapy and RT concomitant with BT. SD: Standard deviation; CC: cervical carcinoma. 
31 (23.7\%) SCC patients. In AC patients, the corresponding figures were 29 (50.9\%), 18 (31.6\%), and $10(17.6 \%)$ cases with a depth of invasion of $1 / 3$, $2 / 3$, and $3 / 3$ of the cervical wall, respectively. With respect to ASC patients, the depth of invasion was $1 / 3$ in 4 cases (28.6\%), $2 / 3$ in 9 cases ( $64.3 \%$ ), and $3 / 3$ in 2 cases ( $7.1 \%)$. There was also a significant difference in LVSI, which was present in 47 (35.9\%) of the SCC patients, $10(17.5 \%)$ of the AC, and 5 (35.7\%) of the ASC patients. There were no differences in the remaining pathological variables (Table 2).

The 5 -year DFP (Fig. 1) was $94.4 \%$ for SCC, $98.1 \%$ for AC, and $92.3 \%$ for ASC cases and did not reach the mean. There were no significant differences $(p=$ 0.55 ) in the DFP between the three histologies. The 5 -year OS (Fig. 2) was $97.9 \%$ in SCC, $97.8 \%$ in AC, and $100 \%$ in ASC cases, with no significant differences ( $p=0.702)$; the mean OS was not reached in any histology.

In the univariate analysis (Table 3 ) of OS, only recurrence exerted an influence on the patient's prognosis (hazard ratio [HR]: $15.5 ; 95 \%$ confidence interval [95\% Cl]: 2.6-93.5; $p=0.003$ ). Regarding the DFP, the following variables were found to influence recurrence: RH Type 3 surgery (HR: 0.24; $95 \%$ $\mathrm{Cl}$ : 0.06-0.096; $\mathrm{p}=0.04)$; the presence of positive margins (HR: 6.7; 95\% Cl: 1.67-26.9; $\mathrm{p}=0.01$ ), and the administration of $\mathrm{BT}$ alone as adjuvant management (HR: $11.1 ; 95 \% \mathrm{Cl}: 1.55-78.5 ; \mathrm{p}=0.02$ ). These variables were included in the multivariate analysis (Table 4), but none were found to independently influence recurrence.

\section{DISCUSSION}

The most common histologies in CC are SCC followed by $A C$, with relative frequencies of $75.8-86 \%$ and $14-24.2 \%$, respectively; the prevalence of AC has been reported to have increased over the past 10 years ${ }^{14}$.

Figure 1. Disease-free period by histology. 5-year diseasefree period of $94.4 \%$ for squamous cell carcinoma, $98.1 \%$ for adenocarcinoma, and $92.3 \%$ for adenosquamous carcinoma $(p=0.557)$.

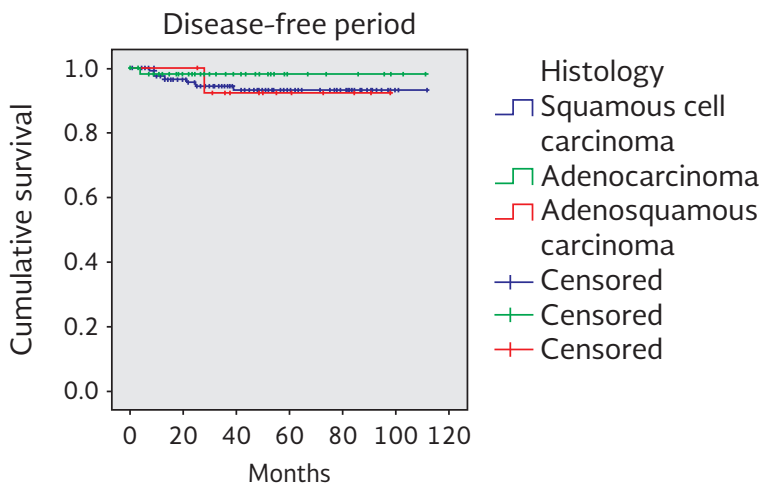

Figure 2. Overall survival by histology. 5-year overall survival of $97.9 \%$ for squamous cell carcinoma, $97.8 \%$ for adenocarcinoma, and $100 \%$ for adenosquamous carcinoma $(p=0.702)$.

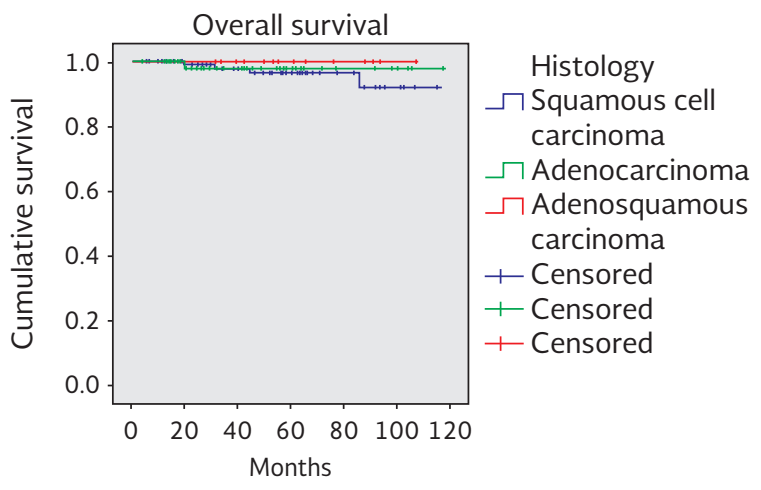

Table 2. Pathological characteristics by histology in patients with early-stage CC who receive a radical hysterectomy

\begin{tabular}{|c|c|c|c|c|}
\hline Characteristics & $\begin{array}{c}\text { Squamous** } \\
131(64.9)\end{array}$ & $\begin{array}{c}A C^{* *} \\
57(28.2) \\
\end{array}$ & $\begin{array}{c}\text { Adenosquamous** } \\
14(6.9)\end{array}$ & $\mathrm{p}$ \\
\hline TS $(\mathrm{cm})^{*}$ & $1.8(1.4)$ & $1.7(0.98)$ & $1.8(0.99)$ & 0.711 \\
\hline \multicolumn{5}{|l|}{ Stromal invasion** } \\
\hline $1 / 3$ & $67(51.1)$ & $29(50.9)$ & $4(28.6)$ & 0.04 \\
\hline $2 / 3$ & $33(25.2)$ & $18(31.6)$ & $9(64.3)$ & \\
\hline $3 / 3$ & $31(23.7)$ & $10(17.6)$ & $2(7.1)$ & \\
\hline LVIS** & $47(35.9)$ & $10(17.5)$ & $5(35.7)$ & 0.04 \\
\hline Positive margins** & $12(9.2)$ & $2(3.5)$ & $1(7.1)$ & 0.397 \\
\hline Positive para-metria** & $5(3.8)$ & $2(3.5)$ & $0(0)$ & 0.759 \\
\hline Positive lymph nodes* & $12(9.2)$ & $3(5.3)$ & $0(0)$ & 0.353 \\
\hline
\end{tabular}

*Mean (SD); **Absolute frequency (percentage).

TS: tumor size; LVIS: Iymphovascular space invasion; SD: standard deviation; CC: cervical carcinoma. 
Table 3. Univariable analysis of patients with early-stage cervical cancer receiving radical hysterectomy

\begin{tabular}{|c|c|c|c|c|c|}
\hline \multirow[t]{2}{*}{ Characteristic } & \multirow[t]{2}{*}{ n (\%) } & \multicolumn{2}{|l|}{ OS } & \multicolumn{2}{|l|}{ DFP } \\
\hline & & $\mathrm{HR}(95 \% \mathrm{Cl})$ & $\mathrm{p}$ & $\mathrm{HR}(95 \% \mathrm{Cl})$ & $\mathrm{p}$ \\
\hline \multicolumn{6}{|l|}{ Age } \\
\hline$\geq 40$ years & $167(82.7)$ & 1 & & 1 & \\
\hline$<40$ years & $35(17.3)$ & $0.03(0.01-242.5)$ & 0.45 & $0.04(0.01-37.82)$ & 0.35 \\
\hline \multicolumn{6}{|l|}{$\mathrm{BMI}$} \\
\hline$\leq 30$ & $144(71.3)$ & 1 & & 1 & \\
\hline$>30$ & $58(28.7)$ & $0.75(0.084-6.77)$ & 0.80 & $0.76(0.16-3.64)$ & 0.73 \\
\hline \multicolumn{6}{|l|}{ Histology } \\
\hline SCC & $131(64.9)$ & 1 & & 1 & \\
\hline$A C$ & $57(28.2)$ & $0.65(0.07-5.83)$ & 0.70 & $0.34(0.04-2.76)$ & 0.31 \\
\hline ASC & $14(6.9)$ & $0.001(0.001-9.00)$ & 0.99 & $1.07(0.13-8.70)$ & 0.95 \\
\hline \multicolumn{6}{|l|}{ Type of surgery } \\
\hline $\mathrm{RH} 2$ & $84(41.1)$ & 1 & & 1 & \\
\hline $\mathrm{RH} 3$ & $119(58.9)$ & $0.47(0.07-3.07)$ & 0.43 & $0.24(0.06-0.96)$ & 0.044 \\
\hline \multicolumn{6}{|c|}{ 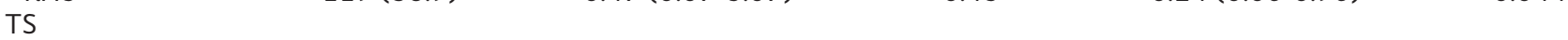 } \\
\hline$<2 \mathrm{~cm}$ & $137(67.8)$ & 1 & & 1 & \\
\hline $2-4 \mathrm{~cm}$ & $52(25.7)$ & $0.69(0.08-6.13)$ & 0.74 & $1.35(0.34-5.38)$ & 0.34 \\
\hline$>4 \mathrm{~cm}$ & $13(6.4)$ & $0.001(0.001-9.00)$ & 0.99 & $0.001(0.001-9.00)$ & 0.99 \\
\hline \multicolumn{6}{|l|}{ Stromal invasion } \\
\hline $1 / 3$ & $100(49.5)$ & 1 & & 1 & \\
\hline $2 / 3$ & $60(29.7)$ & $1.635(0.23-11.69)$ & 0.63 & $0.76(0.14-4.12)$ & 0.75 \\
\hline $3 / 3$ & $42(20.8)$ & $1.22(0.11-13.51)$ & 0.87 & $1.96(0.44-8.80)$ & 0.38 \\
\hline \multicolumn{6}{|l|}{ LVSI } \\
\hline Absent & $140(69.3)$ & 1 & & 1 & \\
\hline Present & $62(30.7)$ & $3.79(0.63-22.81)$ & 0.15 & $2.96(0.79-11.05)$ & 0.11 \\
\hline \multicolumn{6}{|l|}{ Margins } \\
\hline Negative & $187(92.6)$ & 1 & & 1 & \\
\hline Positive & $15(7.4)$ & $2.68(0.29-23.99)$ & 0.38 & $6.70(1.67-26.93)$ & 0.01 \\
\hline \multicolumn{6}{|l|}{ Parametria } \\
\hline Negative & $195(96.5)$ & 1 & & 1 & \\
\hline Positive & $7(3.5)$ & $6.49(0.72-58.32)$ & 0.09 & $3.67(0.462-9.37)$ & 0.22 \\
\hline \multicolumn{6}{|l|}{ Lymph nodes } \\
\hline Negative & $187(92.6)$ & 1 & & 1 & \\
\hline Positive & $15(7.4)$ & $3.42(0.38-30.69)$ & 0.27 & $1.65(0.21-13.19)$ & 0.64 \\
\hline \multicolumn{6}{|l|}{ Adjuvancy } \\
\hline No & $116(57.4)$ & 1 & & 1 & \\
\hline BT & $10(5)$ & $0.01(0.001-9.00)$ & 0.99 & $11.05(1.55-78.45)$ & 0.02 \\
\hline RT & $46(22.8)$ & $1.15(0.10-12.72)$ & 0.91 & $3.87(0.65-23.14)$ & 0.14 \\
\hline Chem/RT & $30(14.9)$ & $4.11(0.57-29.90)$ & 0.16 & $3.88(0.55-27.53)$ & 0.18 \\
\hline Recurrence & $193(95.5)$ & 1 & & & \\
\hline No & $9(4.5)$ & $15.54(2.58-93.53)$ & 0.003 & NA & NA \\
\hline
\end{tabular}

HR: hazard ratio; BMI: body mass index; RH2: radical hysterectomy Type 2; RH3: radical hysterectomy Type 3; SCC: squamous cell carcinoma; AC: adenocarcinoma; ASC: adenosquamous carcinoma; TS: tumor size; DFS: disease-free period; LVSI: Iymphovascular space invasion; BT: brachytherapy; RT: radiotherapy; Chem/RT: chemotherapy and RT concomitant with BT; OS: Overall survival; DFP: disease-free period; HR: Hazard ratio; $\mathrm{Cl}$ : Confidence interval.

Conventionally, early-stage SCC, AC, and ASC should be treated in the same manner, with RH and BPL. In the presence of pathological risk factors for recurrence, adjuvant treatment should be administered with RT and $\mathrm{BT}$, and if high-risk factors are present, adjuvant treatment should be provided with Chem/RT ${ }^{15}$. In our study, ASC had more frequently intermediate risk factors, especially LVIS and stromal invasion of more than $1 / 3$, reason for which the RT and BT predominate in this histological group. In addition, a difference in the high-risk factors between the histologies was not found, and therefore, a difference in the adjuvant handling with Chem/RT is not reflected. Important is to mention that these risk factors are used for adjuvant treatment and are very well established in the literature.

There is some controversy regarding the prognosis and optimal management of patients based on the CC histology. Some studies have reported that AC and 
Table 4. Multivariate analysis of the disease-free period of patients with early-stage CC who receive a radical hysterectomy

\begin{tabular}{lccc}
\hline & $\mathrm{n}(\%)$ & $\mathrm{HR}(95 \% \mathrm{Cl})$ & $\mathrm{p}$ \\
\hline Type of surgery & & & \\
RH2 & $84(41.1)$ & 1 & 0.09 \\
RH3 & $119(58.9)$ & $0.27(0.06-1.26)$ & \\
Margins & & & 0.18 \\
$\quad$ Negative & $187(92.6)$ & 1 & \\
$\quad$ Positive & $15(7.4)$ & $3.27(0.58-18.39)$ & \\
Adjuvant treatment & & & \\
$\quad$ No & $116(57.4)$ & 1 & 0.07 \\
BT & $10(5)$ & $6.89(0.85-55.81)$ & 0.09 \\
RT & $46(22.8)$ & $4.73(0.75-29.95)$ & 0.09 \\
Chem/RT & $30(14.9)$ & $2.26(0.24-21.73)$ & 0.48 \\
\hline
\end{tabular}

HR: hazard ratio; $\mathrm{RH} 2$ : radical hysterectomy Type 2;

$\mathrm{RH} 3$ : radical hysterectomy Type 3; BT: brachytherapy;

$\mathrm{RT}$ : radiotherapy and brachytherapy; Chem/RT: chemotherapy and

radiotherapy concomitant with brachytherapy; HR: hazard ratio;

Cl: confidence interval; CC: cervical carcinoma.

SCC histologies have similar rates of survival, while other studies have demonstrated a difference according to histology. In a study by Park et al., in which the authors analyzed 1200 patients with SCC and AC histologies, 5-year OS rates of $94 \%$ and $90 \%$ were found, respectively, with recurrence rates of $14 \%$ and $9 \%$, respectively, without significant differences ${ }^{9}$. Galic et al., in a SEER analysis of 24,562 patients in the early and advanced stages of CC, compared SCC, AC, and ASC histologies. Comparisons of AC and ASC to SCC in the early stages led to an odds ratio of 1.39 ( $95 \%$ $\mathrm{Cl}: 1.23-1.56)$ for $\mathrm{AC}$ and of 1.55 (95\% Cl: $1.32-$ 1.82) for ASC, which was significant $(p=0.001)^{16}$. The clinical stages of the different histologies could exert an influence on the prognosis of patients with CC. Shingleton et al. found that there was no difference with regard to 5-year survival between SCC and AC histologies in Stage IB ( $p=0.496)$, but there was a difference between Stage II SCC and AC $(p=0.001)^{17}$. In our study, no differences were found in OS or in DFP between SCC, AC, and ASC histologies in patients with $\mathrm{CC}$ in early clinical stages who were submitted to $\mathrm{RH}$ with BPL with or without adjuvant treatment. This finding is consistent with those from other studies that suggest that in the early stages, there is no difference in prognosis among histologies, whereas in locally advanced stages, AC and ASC histologies appear to have a worse response rate to Chem/RT.

The risk factors that were significant in the univariate analysis of the DFP were the presence of positive margins, the administration of BT only, and the type of surgery, with a more radical surgical procedure being a protective factor. These factors did not exert an independent influence but nonetheless could indicate that more extensive surgical procedures can prevent positive margins and that when adjuvant treatment is needed, it should be complete to be effective. With respect to OS, the sole factor that exerted an influence was the presence of recurrence, in particular, if the recurrence was distant.

It is important to emphasize that there was a greater percentage of patients with ASC histology who received adjuvant treatment, in particular, some modality of RT (Table 1 ). This is probably due to the fact that there was a higher percentage of patients with this histology who had more than a third of stromal invasion (71.4\%) and a relatively high percentage of LVSI (35.7\%). Therefore, those who have received some type of adjuvant management in this group of patients can explain, to a certain extent, the compensation in the DFP and OS between the histologies, especially the ASC.

The majority of studies comparing histologies in CC are retrospective studies. Although some studies support the idea that $\mathrm{AC}$ has a worse prognosis, one must consider that on occasion, ASC histology is included in $A C^{5,11,18}$. To the best of our knowledge, this is the first study to compare separately the three most common histologies of CC in the early stages in Mexican women at a single institution, as most of the literature includes only SCC and AC. Similarly, in our study, all the patients with mixed histologies were excluded, and the groups were relatively homogeneous. However, this study also presents certain limitations. First, it was retrospective in nature. It is also noteworthy that there were only a small number of patients with ASC histology. Finally, although adjuvant treatment at the Institution is well standardized, there were several patients who received BT only, and this could have modified the prognosis of these patients.

In conclusion, DFS and OS do not differ between the most common histologies in early-stage CC. To avoid recurrences, it is important to perform surgical procedures that ensure negative margins, and if there are risk factors for recurrence present, adjuvant treatment in its complete form is recommended. The present study is hypothesis generated, which enables the 
understanding of this neoplasm in Mexican women. Thus, the prospective studies should be conducted that evaluate the prognosis of each CC histological type studied herein.

\section{REFERENCES}

1. Ferlay J, Soerjomataram I, Ervik M, et al. GLOBOCAN 2012 v1.0, Cancer Incidence and Mortality Worldwide. Lyon, France: IARC Cancer, Base; 2013. p. 11.

2. Smith HO, Tiffany MF, Qualls CR, Key CR. The rising incidence of adenocarcinoma relative to squamous cell carcinoma of the uterine cervix in the United States-a 24-year population-based study. Gynecol Oncol. 2000;78:97-105.

3. Adegoke O, Kulasingam S, Virnig B. Cervical cancer trends in the United States: A 35-year population-based analysis. J Womens Health (Larchmt). 2012;21:1031-7.

4. Ries LA, Melbert D, Krapcho M. SEER. Cancer Statistics Review, 1975-2004. Bethesda, MD: National Cancer Institute; 2006. Available from: http://www.seer.cancer.gov/csr/1975_2004. [Last accessed on 2015 Oct 03].

5. Irie T, Kigawa J, Minagawa $Y$, et al. Prognosis and clinicopathological characteristics of Ib-llb adenocarcinoma of the uterine cervix in patients who have had radical hysterectomy. Eur J Surg Oncol. 2000;26:464-7

6. Nakanishi T, Ishikawa H, Suzuki $Y$, Inoue T, Nakamura S, Kuzuya K. A comparison of prognoses of pathologic stage Ib adenocarcinoma and squamous cell carcinoma of the uterine cervix. Gynecol Oncol. 2000;79:289-93.

7. Katanyoo K, Sanguanrungsirikul S, Manusirivithaya S. Comparison of treatment outcomes between squamous cell carcinoma and adenocarcinoma in locally advanced cervical cancer. Gynecol Oncol. 2012;125:292-6.
8. Lee YY, Choi CH, Kim TJ, et al. A comparison of pure adenocarcinoma and squamous cell carcinoma of the cervix after radical hysterectomy in stage IB-IIA. Gynecol Oncol. 2011;120: 439-43.

9. Park JY, Kim DY, Kim JH, Kim YM, Kim YT, Nam JH. Outcomes after radical hysterectomy in patients with early-stage adenocarcinoma of uterine cervix. Br J Cancer. 2010;102:1692-8.

10. Ioffe OB. The uterine cervix. In: Silverberg SG, DeLellis RA, Frable WJ, LiVolsi VA, Wick MR, editors. Principles and Practice of Surgical Pathology and Cytopathology. Philadelphia, PA: Churchill Livingstone; 2006. p. 542-95.

11. Kleine W, Rau K, Schwoeorer D, Pfleiderer A. Prognosis of the adenocarcinoma of the cervix uteri: A comparative study. Gynecol Oncol. 1989;35:145-9.

12. Lee KB, Lee JM, Park CY, Lee KB, Cho HY, Ha SY. What is the difference between squamous cell carcinoma and adenocarcinoma of the cervix? A matched case-control study. Int J Gynecol Cancer. 2006;16:1569-73.

13. Wells M, Oslor AG, Crum CP, et al. Tumors of uterine cervix. In: Tavassoli FA, Stratton MR, editors. Pathology and Genetics: Tumours of the Breast and Female Genital Organs. Lyon: WHO; 2003. p. 259-89.

14. Ward KK, Shah NR, Saenz CC, McHale MT, Alvarez EA, Plaxe SC. Changing demographics of cervical cancer in the United States (1973-2008). Gynecol Oncol. 2012;126:330-3.

15. Koh WJ, Greer BE, Abu-Rustum NR, et al. Cervical cancer. J Natl Compr Canc Netw. 2013;11:320-43.

16. Galic V, Herzog TJ, Lewin SN, et al. Prognostic significance of adenocarcinoma histology in women with cervical cancer. Gynecol Oncol. 2012;125:287-91.

17. Shingleton HM, Bell MC, Fremgen A, et al. Is there really a difference in survival of women with squamous cell carcinoma, adenocarcinoma, and adenosquamous cell carcinoma of the cervix? Cancer. 1995;76 10 Suppl:1948-55.

18. Eifel PJ, Burke TW, Morris M, Smith TL. Adenocarcinoma as an independent risk factor for disease recurrence in patients with stage IB cervical carcinoma. Gynecol Oncol. 1995;59:38-44. 